\title{
Atlas of Non-Invasive Coronary Angiography by Multidetector Computed Tomography
}

\author{
G. Pons-Llado and R. Leta-Petracca, eds.
}

New York, NY: Springer, 2006, 129 pages, $\$ 129$

This book is a small atlas divided into 5 chapters with a hard cover and high-quality paper. The editors are from the Cardiology Department of Hospital de la Santa Creu I Sant Pau, University Autonoma de Barcelona, Barcelona, Spain. The chapters are a mix of basic introductory information about coronary CT angiography (CTA) and high-quality illustrations. Specifically, the chapters cover the following topics: performance of cardiac CT, anatomy of the coronaries, calcium scoring, coronary artery stenoses, and functional assessment of heart chambers. The information is presented in a focused, clear, and simple language even for those who are not knowledgeable about coronary CTA. Additionally, the book provides the important facts on how to perform coronary CTA and correctly interpret it. The illustrations are clear and well-labeled, with good legends that follow the text closely and clarify the information. Each page in the book has impressive color or black and white illustrations obtained using coronary CTA, mostly from 3-dimensional displays. I was particularly impressed with how the authors were able to demonstrate the anatomy and variation in all coronary arteries using only 3-dimensional CTA images. Occasionally, the legend is on the page opposite the illustration, creating slight confusion. The references are adequate, and most are recent and from different sources.

Overall, the book can serve as an easy and quick introduction to coronary CTA for beginners in the field. It would also be an excellent teaching aid for physicians in training or before attending a course about coronary CTA. I highly recommend this informative book with impressive illustrations to any clinician interested in learning about coronary CTA.

Isis Gayed University of Texas M.D. Anderson Cancer Center Houston, Texas 